\title{
Digital pulse processing for Nal(Tl) detectors
}

\author{
Authors: A. Di Fulvio, T. H. Shin, M. C. Hamel and S. A. Pozzi \\ Department of Nuclear Engineering and Radiological Science of the University of Michigan, Ann Arbor, 2200 \\ Bonisteel Boulevard, Ann Arbor, Michigan 48109-2104
}

\begin{abstract}
We apply two different post-processing techniques to digital pulses induced by photons in Nal(TI) detector and compare the obtained energy resolution to the standard analog approach. Our digital acquisition approach is performed using a single-stage acquisition with a fast digitizer. Both the postprocessing techniques we propose rely on signal integration. In the first, the pulse integral is calculated by directly numerically integrating the pulse digital samples, while in the second the pulse integral is estimated by a model-based fitting of the pulse. Our study used a $7.62 \mathrm{~cm} \times 7.62 \mathrm{~cm}$ cylindrical Nal(TI) detector that gave a 7.60\% energy resolution (at $662 \mathrm{keV}$ ), using the standard analog acquisition approach, based on pulse shaping amplifier. The new direct numerical integration yielded a $6.52 \%$ energy resolution. The fitting approach yielded a $6.55 \%$ energy resolution, and, although computationally heavier than numerical integration, is preferable when only the early samples of the pulse are available. We also evaluated the timing performance of a fast-slow detection system, encompassing an EJ-309 and a Nal(TI) scintillator. We use two techniques to determine the pulse start time: constant fraction discrimination (CFD) and adaptive noise threshold timing (ANT), for both the analog and digital acquisition approach. With the analog acquisition approach, we found a system time resolution of $5.8 \mathrm{~ns}$ and $7.3 \mathrm{~ns}$, using the constant fraction discrimination and adaptive noise threshold timing, respectively. With the digital acquisition approach, a time resolution of 1.2 ns was achieved using the ANT method and 3.3 ns using CFD at $50 \%$ of the maximum, to select the pulse start time. The proposed direct digital readout and post-processing techniques can improve the application of $\mathrm{Nal}(\mathrm{TI})$ detectors, traditionally considered 'slow', for fast counting and correlation measurements, while maintaining a good measurement of the energy resolution.
\end{abstract}

(C) 2015. This manuscript version is made available under the Elsevier user license http://www.elsevier.com/open-access/userlicense/1.0/ 


\section{Introduction}

The decay time of the scintillation pulse in $\mathrm{Nal}(\mathrm{TI})$ detectors is characterized by a prevailing $\approx 230 \mathrm{~ns}$ component. This time response is relatively slow, compared to organic scintillators and limits the use of $\mathrm{NaI}(\mathrm{TI})$ detectors for fast timing and coincidence counting applications. In this work, we use newly developed, fast, analog-to-digital converters to recover the integral of the current pulse from its few early digital samples, thereby significantly enhancing timing performance, while maintaining energy resolution.

Crystalline sodium iodide, doped with thallium [1], is the detector of choice for routine gamma spectroscopy and is used for a variety of other applications, ranging from nuclear medicine [2] to gamma-ray imaging [3], high energy physics [4], and nuclear safeguards [5]. Nal(TI) has an excellent light yield (38000 photons/MeV), a near linear response in the 10-1000 keV energy range, at a relatively low production cost. The crystal is typically coupled to a photomultiplier tube (PMT). Upon radiation interaction, a light pulse is produced within the impurity-activated crystal and is converted to an electrical pulse by the PMT. When compared to other types of organic and inorganic scintillators (e.g. BGO and activated xylene-naphthalene), Nal(TI) has a poorer time resolution, with an overall pulse length of over $1 \mu$ s [6].

A typical pulse from the anode of a photomultiplier, coupled to a Nal(TI) crystal, reaches its absolute maximum value in 40-80 ns and recovers the baseline with a dominant scintillation decay time of 230 $250 \mathrm{~ns}$ [6]. This signal can either be acquired by means of an analog charge-sensitive amplifier or rapidly sampled and digitized using an analog-to-digital converter. Traditional analog acquisition methods rely on the first approach, where a charge sensitive amplifier is used to integrate the current pulse over time, yielding an overall response time greater or equal to the entire pulse length. In our approach, we use fast digitizers. These devices convert the analog radiation-induced pulse into a digital number with high sampling rates of the order of $\mathrm{GHz}$, without requiring a preamplification stage. We propose two techniques for the analysis of digital signal from Nal(TI) detectors. The first consists of numerically integrating the digital samples of the scintillation pulse, whereas the second estimates the entire pulse integral by fitting a limited number of the pulse samples. The energy resolution performance of these two digital approaches is compared to the standard analog pulse acquisition method. We investigated the timing performance of a two-detector system ( $\mathrm{Nal}(\mathrm{TI})$ and EJ-309) in order to quantify the effect of digital and analog acquisition approach on detection time resolution. We measured the time delay between the detection of two events emitted in coincidence. We used two techniques to estimate the pulse onset time: the standard constant fraction timing and a new technique based on signal-to-noise discrimination. Their application to digital and analog pulses is discussed.

\section{Materials and methods}

A simple irradiation experiment was set up to evaluate energy resolution achieved using the analog and digital approaches. A cylindrical Nal(TI) detector (type 771, with integrated photomultiplier tube 38-B38, distributed by Scionix, NL) having dimensions of $7.62 \mathrm{~cm}$ (diameter) and $7.62 \mathrm{~cm}$ (length) was 
irradiated using a 137-Cs source. The scintillation detector is supplied with a built-in voltage divider type VD 12-E1 (Scionix, NL), with no preamplifier. The PMT high voltage gain was set to $1240 \mathrm{~V}$.

\subsection{Analog acquisition approach}

Traditionally, the electronic readout of radiation detectors is based on a chain of analog devices. The PMT signal output is integrated by a charge sensitive preamplifier, fed to a pulse shaper, and eventually digitized for subsequent storage and analysis. Fig. 1.a shows a schematic of the acquisition chain used in our experiment and Fig1.b shows an example of acquired pulses. The front-end acquisition stage is a NIM compatible shaping amplifier: model MSCF-16 PMT by Mesytec (Mesytec GmbH \& Co., Putzbrunn, $D E)$. The inverting charge sensitive amplifier integrates the charge output of the anode signal from the photomultiplier tube. The voltage difference across the feedback capacitor is directly proportional to the electron charge collected at the PMT anode. The second amplification stage multiplies the signal by a gain of $4.9 \mathrm{mV} / \mathrm{pC}$. The subsequent stage is a pulse shaper with a shaping time constant of $1 \mu \mathrm{s}$. The irradiation was carried out by placing a $0.9 \mu \mathrm{Ci}$ Cs-137 source on the detector front face. The acquisition energy threshold was set to $30 \mathrm{keVee}$. An average of 2000 waveforms per second was recorded.
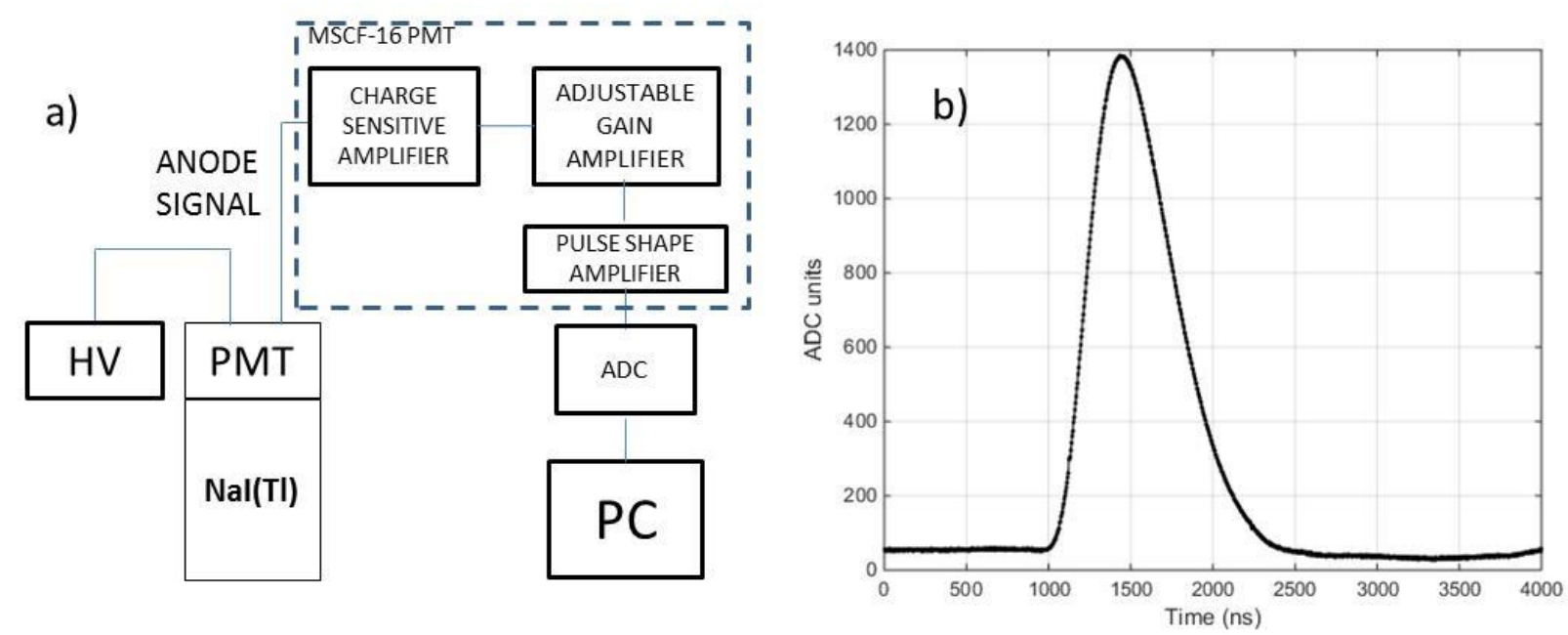

Figure 1. Schematic of the analog acquisition chain (a) and typical shape of gamma-ray generated current pulse in a $\mathrm{Nal}(\mathrm{TI})$ detector, using an intermediate analog shaping amplifier (b).

\subsection{Digital acquisition approach and post-processing algorithms}

The digital signal acquisition approach relies on a fast digitizer (Fig. 2.a). In this study, we used the DT5720 digitizer by CAEN (Viareggio, IT), a 12-bit desktop digitizer with 4 input channels and a $250 \mathrm{MHz}$ sampling frequency [7]. The scintillation detector has an output impedance of $50 \Omega$ for proper matching to the impedance of most frequently used coaxial cables. The input impedance of the digitizer is also $50 \Omega$ to minimize the loading effect and reflection from the load, while maximizing the transferred signal power. The nominal amplitude resolution featured by the digitizer is $0.48 \mathrm{mV}$ within a dynamic range of 
$\pm 1 \mathrm{~V}$. The input signal can be shifted by a software selectable DC offset of an amount up to half of the full range to preserve the entire input signal dynamic range. A minimum voltage headroom, i.e. voltage difference relative to supply rails, of a few hundreds of millivolts is necessary to allow baseline oscillation, which would otherwise cause readout saturation. In this case, we used a DC offset of $920 \mathrm{mV}$, i.e. $80 \mathrm{mV}$ headroom, conservatively chosen for an average signal oscillation of $\pm 2 \mathrm{mV}$ (1 SD) around the baseline. The $1240 \mathrm{~V}$ gain gives a maximum baseline-to-peak voltage amplitude of about $1.2 \mathrm{~V}$ for $662 \mathrm{keV}$ gamma rays. This approach minimized pulse clipping during data acquisition.

The detector was irradiated using a $95 \mu \mathrm{Ci}$ Cs-137 source placed at a distance of $5 \mathrm{~cm}$ from the detector front face. The acquisition trigger threshold was set $45 \mathrm{mV}$ lower than the baseline, corresponding to an electron equivalent recoil energy threshold of $30 \mathrm{keVee}$. An average of $2.4 \times 10^{5}$ waveforms per second was recorded. In these conditions, no dead time is introduced by the acquisition chain, whose maximum data transfer rate is $100 \mathrm{MBps}$. Fig. 2.b shows an example of a digital pulse acquired with the Nal(TI)

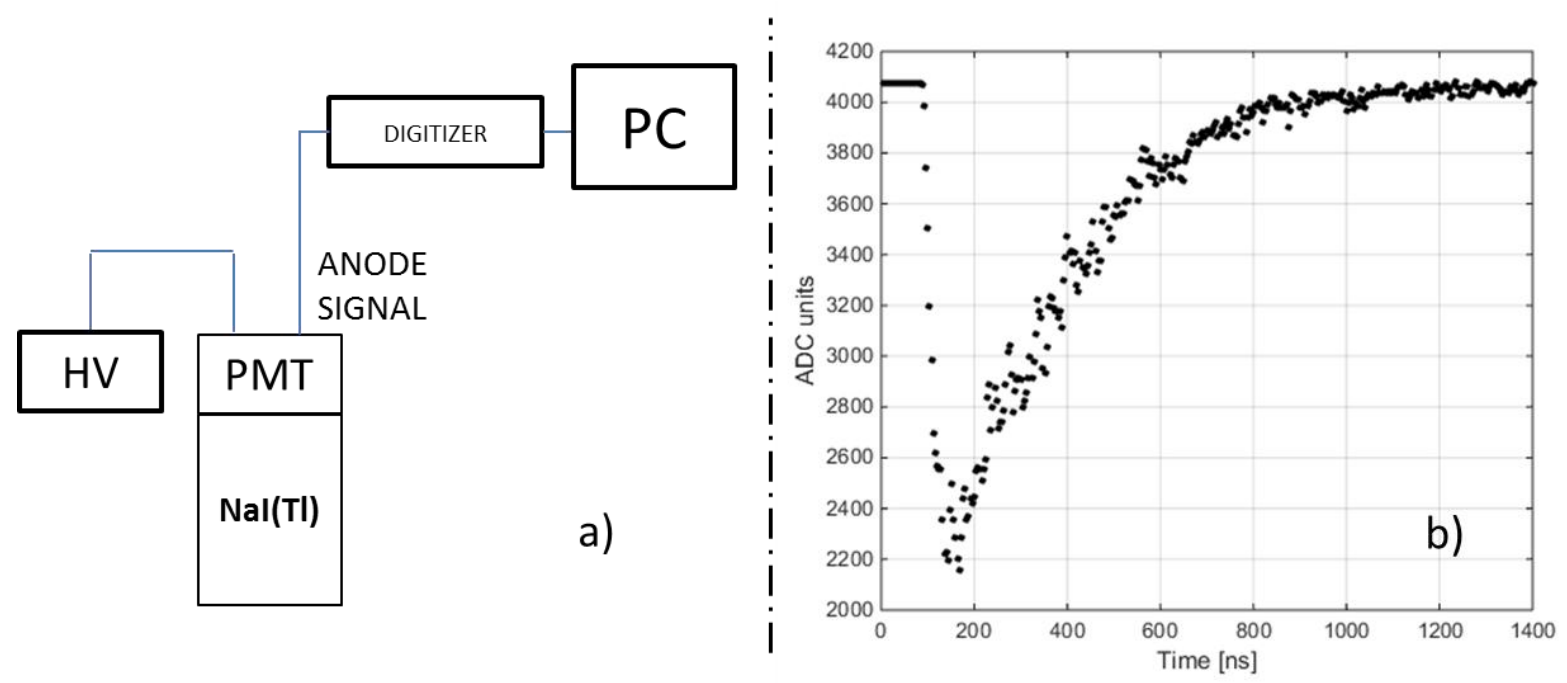

Figure 2. Schematic of the digital acquisition chain (a) and NaI(TI) scintillation pulse after direct analog-to-digital conversion (b).

detector. The digital samples of the pulses generated by 137-Cs gamma rays within the Nal(TI) detector were then processed using the two algorithms described in the following sections.

\section{2.a Numerical integration}

The pulse amplitude of the $\mathrm{Nal}(\mathrm{TI})$ detector pulse shown in Fig. 2.b is in ADC units, i.e. quantization levels in the range $0-\left(2^{N}-1\right)$. In the present case $N=12$, number of nominal bits. The first pulse-processing step consists in inverting the digitized waveforms and subtracting the baseline. The single pulse baseline mean value is calculated by averaging 20 samples before the pulse starting point. The baseline value is subtracted point by point to the signal. This procedure yields a zero-baseline rising edge pulse, its pulse amplitude and integral are thus proportional to the energy released within the detectors. Then, the 
samples in $A D C$ units $\left(\mathrm{V}_{\mathrm{ADC}}\right)$ are converted in Volts $\left(\mathrm{V}_{\mathrm{o}}\right)$ by multiplying the voltage in ADC units by the $A D C$ least significative bit, given by the full scale (FS, $2 \mathrm{~V}$ in this case) divided by the quantization levels, as in Eq. 1.

$V_{o}=V_{A D C}\left(\frac{F S}{2^{N}-1}\right)$

Fig. 3 shows two examples of inverted pulses. The numerical integral of this signal is then calculated by summing $n$ pulse samples, starting from the beginning of the pulse, with $n$ varying from 80 to 360 (entire pulse).

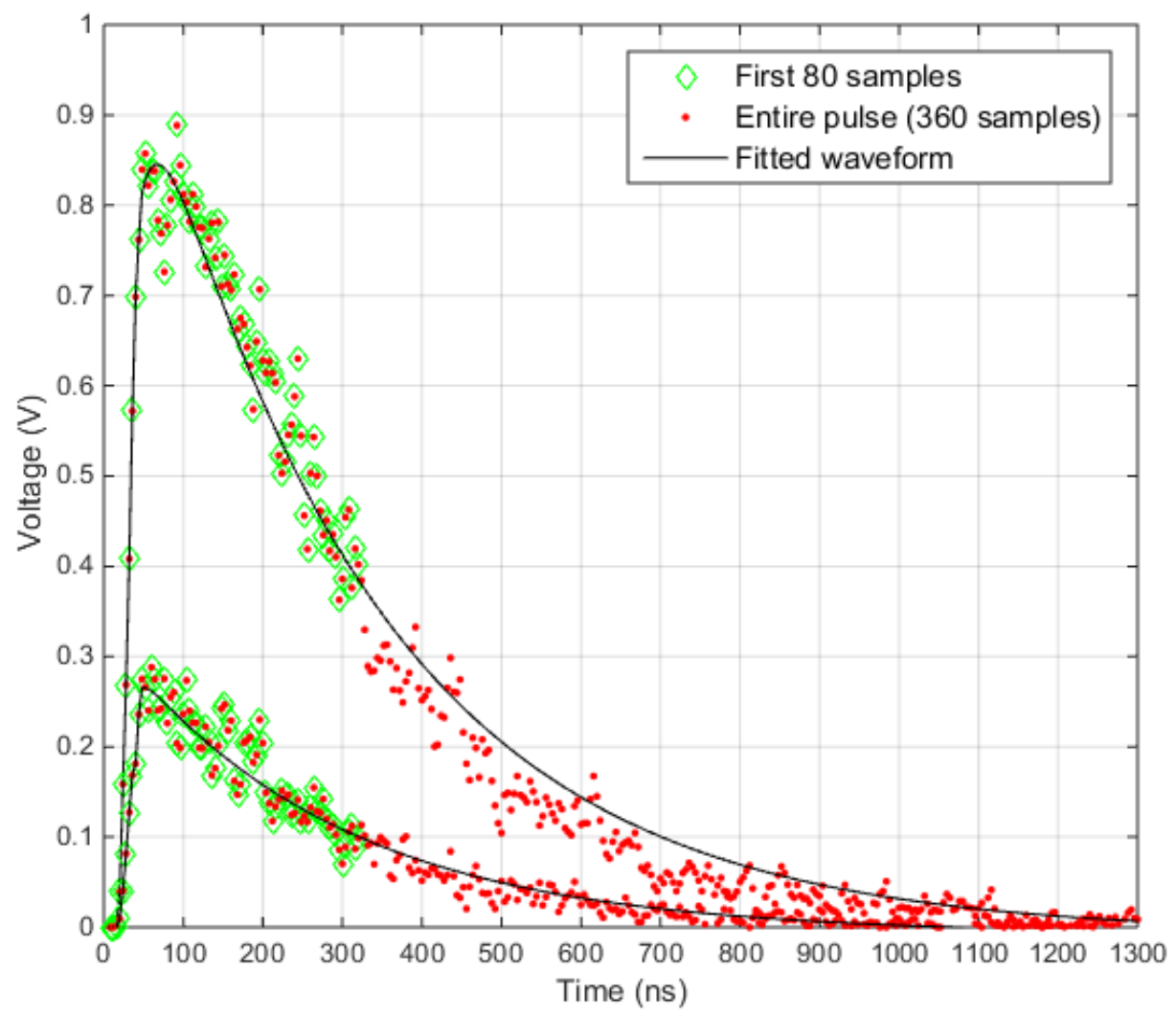

Figure 3. Two digitized Nal(TI) scintillation pulses, compared to their respective fitting function (solid line). Both the measured and the fitted signal have been inverted and baseline subtracted.

\section{2.b Pulse fitting algorithm}

Several approaches have been proposed to model scintillation pulses to describe and quantify some of the physical phenomena behind the complex process of scintillation and photoelectron conversion. 
Conventional single-exponential and bi-exponential fitting functions [8] model the timing distribution of the baseline corrected scintillation pulse as an exponential function with one or two characteristic decay constants, respectively. A bi-exponential model of the pulse is used in this work. The function in Eq. 2 was used to fit the digitized scintillation pulse shown in Fig. 2.b. Two scintillation time constants of the light pulse are explained in Eq. 2: a fast leading edge (second term, $t_{1}$ ) followed by an exponential decay (third term, t2). $A$ is the baseline level, $B$ is well approximated by the pulse minimum value and $C=g$ $(A-B), g$ is an optimization parameter, which varies between 1 and 2 and is optimized the end of the fitting procedure by minimizing the error between the integral of the measured pulse and the fitted one.

$y(t)=A+B \exp \left(-\frac{t}{t_{1}}\right)-C \exp \left(-\frac{t-t_{M I N}}{t_{2}}\right)$

Eq. 2 can also be obtained by a multiparameter convolution-based Nal(TI) pulse model, if the time response of the photomultiplier and excitement of activator centers are disregarded [9]. The simplified version was chosen in this case because the algorithm execution is faster compared to the multiparameter convolution model, while maintaining a good fitting accuracy. The simplified fitting algorithm splits the signal in two parts $\mathrm{y}_{1}$ and $\mathrm{y}_{2}$, of time length $\Delta t_{1}$ and $\Delta t_{2}$ respectively. The segments

are divided by the pulse minimum: $Y_{\text {MIN }} @ t_{\text {MIN }}$. A simple linearization process is used to calculate $t_{1}$ and $t_{2}$, as in Eq. 3, where $Y_{0}$ and $Y_{2}$ are the first and the last pulse samples, respectively.

$t_{1}=\frac{\Delta t_{1}}{\ln ^{Y_{0}} / Y_{M I N}} ; t_{2}=\frac{\Delta t_{2}}{\ln ^{Y_{M I N} / Y_{2}}}$

$t_{1}$ and $t_{2}$ need to be optimized when the pulse amplitude does not reach $1 /$ e of the maximum value within the recorded number of samples. This is usually the case of slow, low-amplitude pulses, which minimally affect the energy resolution at $662 \mathrm{keV}$. The optimization consists in iteratively varying $t_{1}$ and $t_{2}$ in the range between 0.1-10 ns and 200-265 ns respectively, using a bisection algorithm, until the relative error between the integral of the measured samples and the integral of the fitted pulse reaches a minimum.

Fig. 3 shows an example of the results obtained using the fitting algorithm, implemented in Matlab environment [10], for two pulses of different amplitude. The algorithm was tested on an increasing number of samples of the same pulse. The results shown in Fig. 3 were derived using the first 80 samples of the two scintillation pulses, corresponding to $320 \mathrm{~ns}$.

\subsection{Coincidence technique for time resolution measurement}

The analog and digital acquisition chains were compared in terms of time resolution using a version of the delayed-coincidence technique [11]. The general method relies on the measurement of a known time delay between the formation and decay of a nuclear state. In this case, a $0.5 \mu \mathrm{Ci} \mathrm{Na-22} \mathrm{source} \mathrm{was}$ used and two photons, resulting from beta minus decay and subsequent electron-positron annihilation, 
were measured in coincidence. Two detectors: a Nal(TI) $(7.62 \mathrm{~cm} \Phi \times 7.62 \mathrm{~cm})$ and an organic scintillator (EJ-309 $7.62 \mathrm{~cm} \Phi \times 7.62 \mathrm{~cm}$ ) were used. The detectors were placed in front of each other at a distance of $20 \mathrm{~cm}$. The source was placed in-axis with the detectors at $10 \mathrm{~cm}$ distance from each front face. The experiment was performed using both the analog (Fig. 1.a) and the fully digital (Fig. 2.a) acquisition chains. The time resolution of a timing system is determined by time fluctuations in the three main stages of the radiation detection process: radiation interaction with the detection medium, photoelectron generation and transport in the PMT, and pulse conditioning by the electronic acquisition chain. Several authors reviewed the effect of the sources of timing uncertainty on the overall time resolution both experimentally [11] and analytically [12]. The figure of merit of a timing system is usually characterized by the full width at half maximum (FWHM) of the distribution of time delays between the detection of prompt events. Our measured time distribution has $0.3 \mathrm{~ns}$ bin size. We derived the FWHM by spline interpolating the discrete time distribution to find the time corresponding to half of maximum of time distribution. FWHM provides an estimate of the system time resolution and is also related to the standard deviation of a time distribution normally distributed: $\sigma_{t} F W H M=2.355 \sigma_{t}$. The probability distribution of time delays between the detection of two annihilation photons has a variance $\sigma_{t}{ }^{2}$, which depends on the time response of the single detection chain (Eq. 4) [8][11].

$\sigma_{t}^{2}=\sigma_{1 t}^{2}+\sigma_{2 t}^{2}$

In Eq. 4, $\sigma_{1 t}$ and $\sigma_{2 t}$ are the standard deviations of the detection time distributions in each of the two detectors in the coincidence system. In a symmetric two-detector system, the measurement of the FWHM can be used to estimate the time resolution of a single detector.

The time resolution of a scintillator-based measurement system also depends on how the pulse start time is determined. In this work, we used two different methods for determining the pulse start time: constant fraction discrimination (CFD) and adaptive noise threshold (ANT). CFD method locates the first pulse timestamp at a given fraction of the pulse maximum; this approach does not depend on the absolute value of the pulse height. In the ANT approach, the pulse start time is set by the first sample that exceeds the baseline value by a statistically meaningful threshold. The latter is set to four times the standard deviation calculated on the first 20 baseline samples to achieve a signal-to-noise ratio $\geq 24 \mathrm{~dB}$. In both cases, a cubic interpolation was implemented between measured start samples to improve start time estimation accuracy. A cubic interpolation was used to take into account of the early pulse rising edge. This approach is independent of the pulse height, being only affected by the intrinsic noise of the detector and the acquisition chain. 


\section{Results and discussion}

\subsection{Energy resolution}

We used the two integration methods described in Sections 2.2.a and 2.2.b to post process the digitized pulses and estimate the measured energy released by Cs-137 decay photons within the Nal(TI) detector. We evaluated the reliability of the fitting-based approach by comparing the relative error of the pulse integral calculated with the fitted function using 80 samples, with respect to the pulse integral obtained

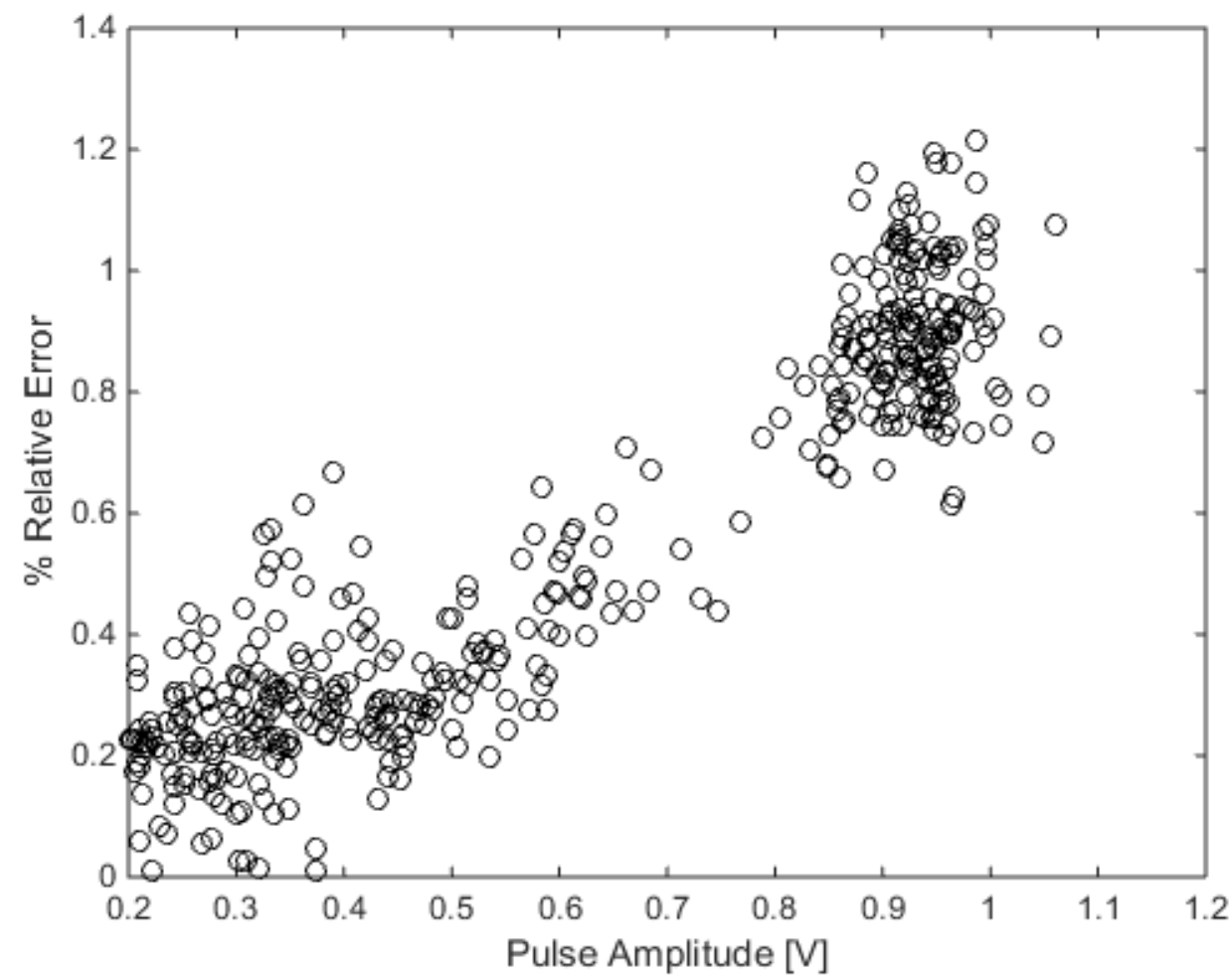

Figure 4. Relative error between the pulse integral calculated on 80 samples using the fitted function and the pulse integral obtained from the numerical integral of the entire measured signal.

from the numerical integral of the entire measured signal, 360 samples. The latter is considered the gold standard. Fig. 4 shows the error for 1000 pulses as a function of the pulse amplitude. The error is below $1.2 \%$ over the experimental voltage range of interest. The results show an error increase with pulse amplitude, the effect is most likely due to a slower pulse decay component [6], which is more relevant at higher photon energies and is not captured by the current model.

Fig. 5 shows Cs-137 spectra calculated using the two computational methods applied to the digitized PMT signal and the analog approach. The spectrum was also obtained as the distribution of the pulse heights, i.e. the peak amplitude of each pulse. The spectral energy resolution is conventionally calculated as the full width at half maximum of the detector peak response to photons of a given energy, divided by the average energy value [8]. Our analog acquisition yielded an energy resolution of 7.6\%. This result is consistent with $\mathrm{Nal}(\mathrm{Tl})$ energy resolution estimates in similar irradiation conditions (i.e. Cs-137 and analog acquisition chain), which range from $8.2 \%$ for large modules $(50.8 \mathrm{~cm}$ length, 
$20.3 \mathrm{~cm}$ diameter) [13] to $6.3 \%$ for smaller crystals (5.08 cm length, $5.08 \mathrm{~cm}$ diameter) [14]. The digital numerical integral approach yielded a $6.63 \%$ energy resolution, using 360 pulse samples. The integral of the fitted pulse, computed on 80 samples, yields a $7.15 \%$ energy resolution. This value is lower than the resolution one would obtain by numerically integrating the same number of pulse samples (7.61\%). Although the fitting algorithm may offer an advantage over the numerical integration algorithm if only the early samples of the pulse are available, the fitting computational time is about 10 times higher than numerical integration. Table 1 summarizes the performance of the proposed methods in terms of energy resolution; the digital approaches are evaluated as a function of the number of processed samples in the pulse.

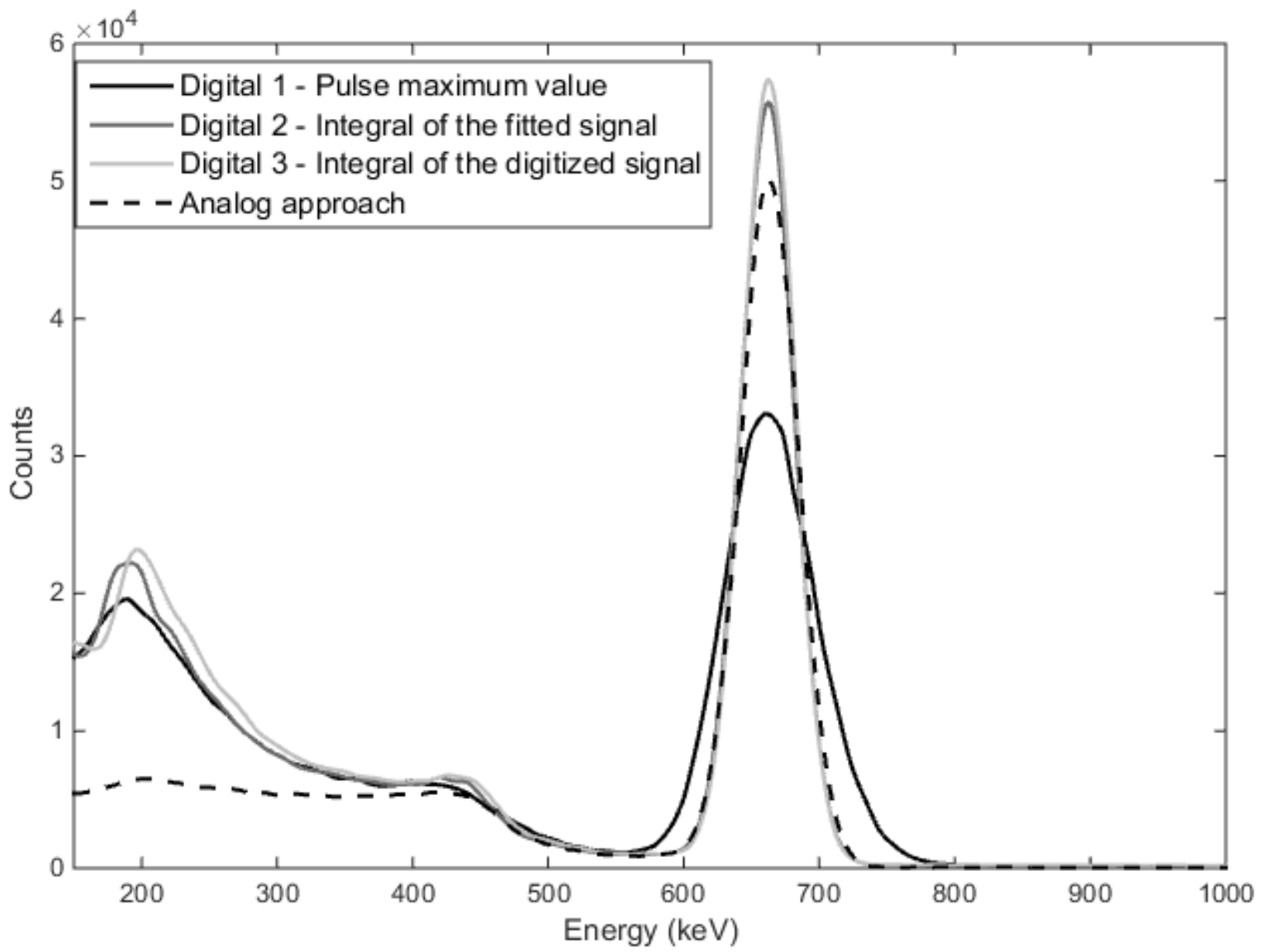

Figure 5. Cs-137 energy spectrum obtained estimating the energy released with the Nal(TI) crystal using the analog and digital approaches. Three different quantities are measured from the digitized pulse: the pulse maximum, the numerical integral of the whole pulse (360 samples, i.e. $1.44 \mu \mathrm{s}$ ) and integral of the fitted pulse obtained using 80 samples. 
Table 1. Energy resolution comparison chart.

\begin{tabular}{|c|c|c|c|c|c|c|c|c|c|}
\hline \# samples & $\begin{array}{c}1 \text { (pulse } \\
\text { maximum) }\end{array}$ & 80 & 120 & 160 & 200 & 240 & 280 & 320 & 360 \\
\hline time (ns) & n.a. & 320 & 480 & 640 & 800 & 960 & 1120 & 1280 & 1440 \\
\hline \% Resolution - Analog & \multicolumn{9}{|c|}{7.60} \\
\hline $\begin{array}{c}\text { \% Resolution - } \\
\text { numerical integral }\end{array}$ & 12.7 & 7.61 & 7.35 & 7.15 & 7.00 & 6.87 & 6.74 & 6.63 & 6.52 \\
\hline \%Resolution - integral of & & 7.15 & 7.09 & 7.07 & 7.04 & 6.99 & 6.84 & 6.58 & 6.55 \\
\hline the fitted pulse ( $\pm 1 S D$ ) & & \pm .08 & \pm .07 & \pm .05 & \pm .08 & \pm .09 & \pm .09 & \pm .09 & \pm .07 \\
\hline
\end{tabular}

\subsection{Time resolution}

The distribution of time delay of a $\mathrm{NaI}(\mathrm{TI})$ and a EJ-309 liquid organic scintillator in detecting annihilation photons emitted in coincidence by a Na-22 source is shown in Fig. 6 . The measurement of the time dispersion around zero of two coincidence events gives an estimate of the time resolution of the overall detection system (i.e. detector, PMT, and acquisition electronics). The use of two different detectors yields an asymmetric distribution of delay times. Our results show that time resolution greatly improves in the digital acquisition mode (Fig. 6.a), as in this case the system time constant is not set by the shaping stage.

We used two methods to estimate the detection time resolution: constant fraction discrimination and adaptive noise threshold. For analog pulses, Fig 6 shows that constant fraction discrimination with threshold at 50\% yields a FWHM of 5.8 ns, lower than ANT (FWHM 7.3 ns). Conversely, for digital pulses, ANT and 50\% CFD yield 1.2 ns and 3.3 ns FWHM, respectively. CFD and ANT are compared in Table 2, for both the analog and digital acquisition approach. CFD should be used when the baseline noise is not negligible, as in the case of the preamplified signal (root-mean-square of the noise voltage signal, $V_{\mathrm{RMS}} 5 \mathrm{mV}$ ). Conversely, the adaptive noise threshold timing performs better than CFD when applied to directly digitized pulses with an oscillation around the mean value steady and low, i.e. $V_{\text {RMS }} 1 \mathrm{mV}$. Importantly, the ANT approach can be implemented in real-time during pulse processing, even before the pulse maximum is acquired.

Poitrasson-Riviere and colleagues [3] performed a coincidence experiment similar to ours using two EJ-309 scintillators and found a FWHM of the time distribution of $1.2 \mathrm{~ns}$, using the CFD method with threshold at $50 \%$ of the pulse maximum, on digitized pulses. We measured a $3.3 \mathrm{~ns}$ time resolution using the same method, i.e. direct digital acquisition and 50\% CFD, but using two different detectors: one organic (EJ-309) and one Nal(TI) scintillator. Using Eq. 4 and assuming two independent detection events, an overall time resolution of approximately $3.0 \mathrm{~ns}$ can be ascribed to the $\mathrm{NaI}(\mathrm{TI})$ detector. 

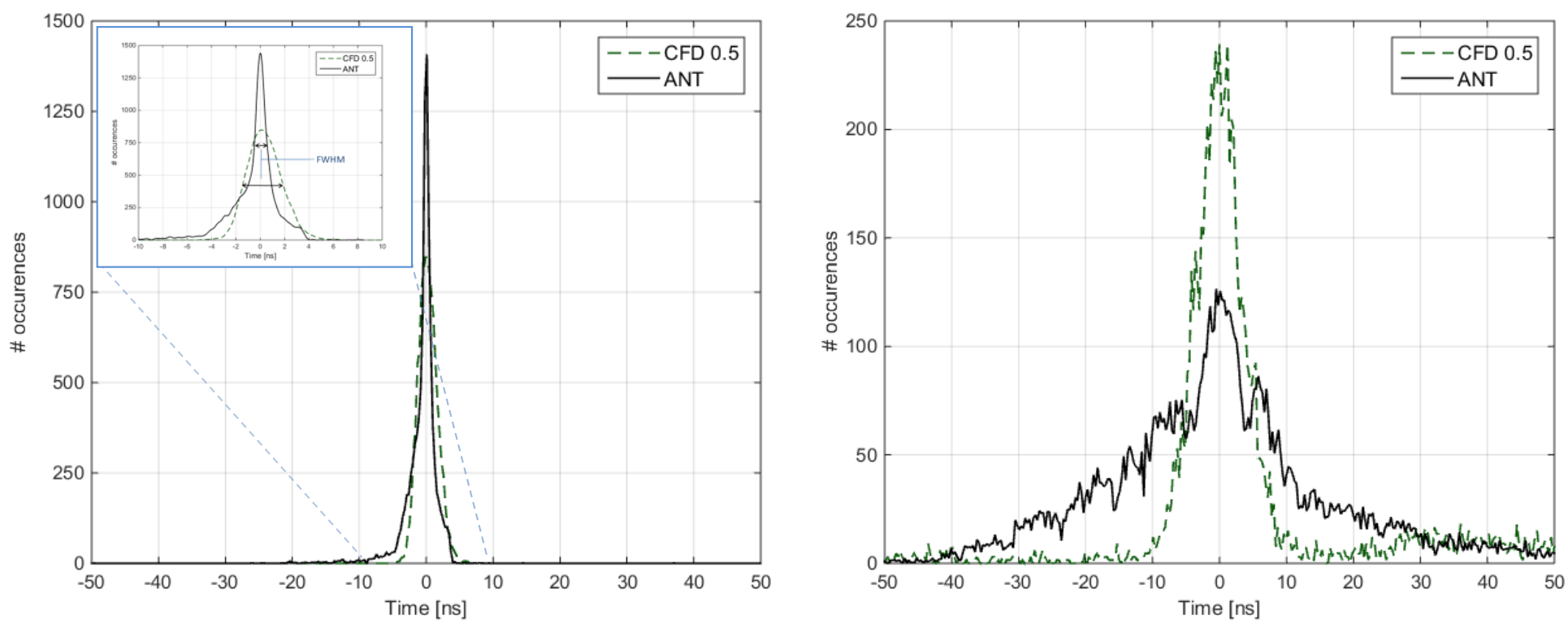

Figure 6. Time detection delay between one $\mathrm{NaI}(\mathrm{TI})$ and one EJ-309 scintillator irradiated using a Na-22 source. Time distributions are shown for a digital (a) and analog (b) acquisition chains.

Table 2. Full width at half maximum characterizing the analog and digital acquisition chains. Two timing derivation methods (CFD@0.5 and SNR discrimination) are also compared. Literature data for EJ-309 organic scintillator are reported for comparison [3].

\begin{tabular}{lccc}
\hline & $\begin{array}{c}\text { FWHM ANT } \\
\text { EJ-309 Nal(TI) (ns) }\end{array}$ & $\begin{array}{c}\text { FWHM CFD 0.5 } \\
\text { EJ-309 Nal(TI) (ns) }\end{array}$ & $\begin{array}{c}\text { FWHM CFD 0.5 } \\
\text { EJ-309 EJ-309 (ns) }\end{array}$ \\
\hline $\begin{array}{l}\text { Analog } \\
\text { approach }\end{array}$ & 7.3 & 5.8 & n.a. \\
$\begin{array}{l}\text { Digital } \\
\text { approach }\end{array}$ & 1.2 & 3.3 & 1.2 \\
\hline
\end{tabular}

\section{Conclusions}

We applied two post-processing techniques to fast digitized pulses produced by a Nal(TI) based detector (crystal size: $7.62 \mathrm{~cm}(\Phi) \times 7.62 \mathrm{~cm}$ ) and compared these approaches to the standard analog acquisition strategy. Both of the proposed digital processing techniques calculate the integral of the digitized pulse, which is a good estimator of the energy released by gamma rays within the Nal(TI) crystal. The first digital technique uses the numerical integral of the pulse. With this method, we found an energy resolution of $6.52 \%$ for 137 -Cs $662 \mathrm{keV}$ gamma rays, compared to $7.60 \%$ in the analog case. The pulse integral can also be recovered using a model-based fitting algorithm. The algorithm is able to recover the entire pulse shape from some measured parameters, i.e. pulse time constants and maximum amplitude. The algorithm can estimate these parameters using only the early part of the scintillation pulse. The integral of one third of the entire pulse, i.e. 320 ns out of $1.44 \mu$ s (entire pulse), was 
estimated using the fitting algorithm, which provided an improvement in resolution $(7.15 \%)$ when compared to the integration approach applied to the same pulse fraction (7.61\%). The fitting method can thus be useful if the whole pulse is not available, i.e. in case of high-throughput, short acquisition time window applications. We also estimated the time resolution of a two-scintillator system, operated in coincidence, using two different methods to select the pulse start time. For pulses acquired using the analog approach, the lowest time resolution ( $5.8 \mathrm{~ns}$ ) was achieved selecting the pulse onset at half of the pulse maximum. Conversely, for directly digitized pulses a time resolution as low as 1.2 ns was obtained selecting as pulse start time the first sample that exceeds the baseline noise by a statistically meaningful threshold. According to our results, the signal characteristics and in particular the signal-tonoise ratio should drive the choice of the method chosen to set the pulse onset.

Direct digital PMT readout is a viable alternative to $\mathrm{Nal}(\mathrm{TI})$ conventional analog readout. The digital approach guarantees superior timing capabilities, while maintaining an adequate if not superior spectroscopic response.

\section{Acknowledgements}

This work was funded in-part by the Consortium for Verification Technology under the Department of Energy National Nuclear Security Administration, award number DE-NA0002534.

\section{References}

[1] R. Hofstadter, Thallium Halide Crystal Counter, Phys. Rev. 72 (1947) 1120-1121. doi:10.1103/PhysRev.72.1120.

[2] D.P. McElroy, L.R. MacDonald, F.J. Beekman, B.E. Patt, J.S. Iwanczyk, B.M.W. Tsui, et al., Performance evaluation of A-SPECT: a high resolution desktop pinhole SPECT system for imaging small animals, IEEE Trans. Nucl. Sci. 49 (2002) 2139-2147. doi:10.1109/TNS.2002.803801.

[3] A. Poitrasson-Rivière, M.C. Hamel, J.K. Polack, M. Flaska, S.D. Clarke, S.A. Pozzi, Dual-particle imaging system based on simultaneous detection of photon and neutron collision events, Nucl. Instruments Methods Phys. Res. Sect. A Accel. Spectrometers, Detect. Assoc. Equip. 760 (2014) 40-45. doi:10.1016/j.nima.2014.05.056. 
[4] S. Akkoyun, A. Algora, B. Alikhani, F. Ameil, G. de Angelis, L. Arnold, et al., AGATA-Advanced GAmma Tracking Array, Nucl. Instruments Methods Phys. Res. Sect. A Accel. Spectrometers, Detect. Assoc. Equip. 668 (2012) 26-58. doi:10.1016/j.nima.2011.11.081.

[5] W. Zhang, J. Yi, P. Mekarski, K. Ungar, B. Hauck, G.H. Kramer, A gamma-gamma coincidence spectrometric method for rapid characterization of uranium isotopic fingerprints, J. Radioanal. Nucl. Chem. 288 (2010) 43-47. doi:10.1007/s10967-010-0868-y.

[6] J.B. Birks, The Theory and Practice of Scintillation Counting, Pergamon Press, 1964.

[7] T. CAEN, User Manual UM2792, (2014).

[8] G.F. Knoll, Radiation detection and measurement, Fourth, Hoboken, N.J. : John Wiley, c2010., 2010.

[9] Y. Shao, A new timing model for calculating the intrinsic timing resolution of a scintillator detector., Phys. Med. Biol. 52 (2007) 1103-17. doi:10.1088/0031-9155/52/4/016.

[10] The MathWorks Inc., MATLAB R2014b, (2014).

[11] M. Moszyński, B. Bengtson, Status of timing with plastic scintillation detectors, Nucl. Instruments Methods. 158 (1979) 1-31. doi:10.1016/S0029-554X(79)90170-8.

[12] S.E. Derenzo, W.-S. Choong, W.W. Moses, Fundamental limits of scintillation detector timing precision., Phys. Med. Biol. 59 (2014) 3261-86. doi:10.1088/0031-9155/59/13/3261.

[13] B.C. Rasco, A. Fijałkowska, M. Karny, K.P. Rykaczewski, M. Wolińska-Cichocka, R. Grzywacz, et al., The nonlinear light output of Nal(TI) detectors in the Modular Total Absorption Spectrometer, Nucl. Instruments Methods Phys. Res. Sect. A Accel. Spectrometers, Detect. Assoc. Equip. 788 (2015) 137-145. doi:10.1016/j.nima.2015.03.087.

[14] X. Wen, H. Yang, Study on a digital pulse processing algorithm based on template-matching for high-throughput spectroscopy, Nucl. Instruments Methods Phys. Res. Sect. A Accel. Spectrometers, Detect. Assoc. Equip. 784 (2015) 269-273. doi:10.1016/j.nima.2014.11.008. 